\title{
Phase 2 study of circulating microRNA biomarkers in castration-resistant prostate cancer
}

Hui-Ming Lin ${ }^{1,2}$, Kate L Mahon 1,2,3,4, Calan Spielman ${ }^{1}$, Howard Gurney, ${ }^{5,6}$, Girish Mallesara ${ }^{6,7}$, Martin R Stockler ${ }^{3,4,6,8,9}$, Patricia Bastick ${ }^{6,10}$, Karen Briscoe ${ }^{6,11}$, Gavin Marx ${ }^{6,12}$, Alexander Swarbrick ${ }^{1,2}$ and Lisa G Horvath ${ }^{*}, 1,3,3,4,6,8$

${ }^{1}$ Cancer Division, The Kinghorn Cancer Centre/Garvan Institute of Medical Research, Darlinghurst, New South Wales 2010, Australia; ${ }^{2}$ St Vincent's Clinical School, Faculty of Medicine, The University of New South Wales, Darlinghurst, New South Wales 2010, Australia; ${ }^{3}$ Department of Medical Oncology, Chris O'Brien Lifehouse, Camperdown, New South Wales 2050, Australia; ${ }^{4}$ Royal Prince Alfred Hospital, Camperdown, New South Wales 2050, Australia; ${ }^{5}$ Crown Princess Mary Cancer Centre, Westmead Hospital, Westmead, New South Wales 2145, Australia; ${ }^{\circ}$ Pharmacogenomics Research for Individualised Medicine (PRIMe) consortium, New South Wales, Australia; ${ }^{7}$ Calvary Mater Newcastle Hospital, Waratah, New South Wales 2298, Australia; ${ }^{8}$ Sydney Medical School, University of Sydney, Camperdown, New South Wales 2050, Australia; ${ }^{9}$ Concord Repatriation General Hospital, Concord, New South Wales 2139, Australia; ${ }^{10}$ St George Hospital, Kogarah, New South Wales 2217, Australia; ${ }^{11}$ Mid North Coast Cancer Institute, Coffs Harbour Health Campus, Coffs Harbour, New South Wales 2450, Australia and ${ }^{12}$ Sydney Adventist Hospital, Wahroonga, New South Wales 2076, Australia

Background: Biomarkers of therapeutic response and prognosis are needed to assist in the sequencing of treatments for metastatic castration-resistant prostate cancer (CRPC). Previously in a Phase 1 discovery study, we identified 14 circulating microRNAs that were associated with response to docetaxel chemotherapy or overall survival. We performed a Phase 2 validation study to verify these findings.

Methods: Using real-time PCR, the levels of the 14 microRNAs were measured in plasma collected before and after the first cycle of docetaxel from a Phase 2 cohort of 89 patients.

Results: The microRNAs were not associated with docetaxel response in the Phase 2 cohort. Higher baseline levels of six microRNAs, predominantly of the miR-200 family, were confirmed to be associated with shorter overall survival. A microRNA signature comprising these six microRNAs predicted high-risk patients in the Phase 2 cohort with a hazard ratio of $4.12(95 \% \mathrm{Cl}$ 2.20-7.70, $P=0.000001$ ). The signature was an independent predictor in multivariable analysis with clinicopathological factors.

Conclusions: The association of circulating microRNAs with overall survival suggests their involvement in CRPC progression.

Prostate cancer is the second most common cancer in men, and the fifth leading cause of cancer death worldwide (Torre et al, 2015). Metastatic prostate cancer is initially sensitive to hormonal manipulation. Resistance to androgen deprivation therapy ultimately occurs with the development of castration-resistant prostate cancer (CRPC). For more than a decade, docetaxel chemotherapy has been the main cytotoxic treatment for CRPC, given its survival and palliative benefits (Petrylak et al, 2004;

*Correspondence: Professor LG Horvath; E-mail: lisa.horvath@lh.org.au

Received 5 December 2016; revised 1 February 2017; accepted 6 February 2017; published online 9 March 2017

(C) 2017 Cancer Research UK. All rights reserved 0007 - 0920/17 
Tannock et al, 2004). However, the response rate is only $50 \%$ (Petrylak et al, 2004; Tannock et al, 2004). In recent years, new therapies for CRPC have emerged, such as cabazitaxel, abiraterone, and enzalutamide (Bishr and Saad, 2013; Wong et al, 2014). Similar to docetaxel, response to these new therapies are variable, and responders eventually develop resistance (Petrylak et al, 2004; Tannock et al, 2004; Ferraldeschi et al, 2015). Therefore, there is an urgent need for novel biomarkers to predict therapeutic response earlier without subjecting non-responders to unnecessary toxicity, and to assist in the sequencing of treatments.

MicroRNAs are short non-coding RNAs (18-25 nucleotides) that negatively regulate gene expression by binding to messenger RNAs (mRNAs) to prevent their translation into protein (Lujambio and Lowe, 2012). Binding occurs at a complementary 'seed' sequence on the $3^{\prime}$ untranslated region of the target mRNA, and a RNA-induced silencing complex is formed with Argonaute proteins, resulting in mRNA cleavage or inhibition of translation. A single microRNA can target hundreds of mRNAs due to imperfect base pairing, and over a thousand microRNAs have been identified in humans (Kozomara and Griffiths-Jones, 2011), thus indicating their importance in regulating cell biology. Indeed, various microRNAs have been implicated in tumour progression and drug resistance (Lujambio and Lowe, 2012). MicroRNAs can be detected in blood as they are secreted by cells, presumably as a means to influence other cells (Turchinovich et al, 2013). Cancer patients have abnormal circulating microRNA profiles, thus raising the possibility of microRNAs as prognostic or therapeutic response biomarkers (Sita-Lumsden et al, 2013; Fabris et al, 2016).

The association of microRNAs with prostate cancer has been extensively reviewed elsewhere (Thieu et al, 2014; Fabris et al, 2016; Fendler et al, 2016). Studies of tumours or biofluids of patients have resulted in the identification of several microRNAs as potential biomarkers of prostate cancer. However, only a few microRNAs such as miR-21, miR-221/222, and miR-375, show consistent associations among these studies. The levels of these three microRNAs were elevated in tumours and/or biofluids of prostate cancer patients $v s$ healthy controls. These microRNAs were also elevated in tumours and/or biofluids of patients with metastatic compared to those with localised prostate cancer, where high levels of miR-375 was associated with poor survival in CRPC (Thieu et al, 2014; Fabris et al, 2016; Fendler et al, 2016). Only a few studies have investigated the potential of circulating microRNAs as therapeutic response biomarkers in CRPC (Gonzales et al, 2011; Zhang et al, 2011; Cheng et al, 2013). These studies reported that circulating levels of miR-141, miR-210 or miR-21 in CRPC patients were associated with response to treatment such as docetaxel and mitoxantrone. However, these studies were limited by cohort size and lack of validation.

Previously, our group identified circulating microRNAs associated with docetaxel response or overall survival in CRPC in a Phase 1 biomarker discovery study (Lin et al, 2014; Figure 1). The baseline levels or post-docetaxel change in the levels of six microRNAs (miR-20a, miR-145a, miR-200b, miR-200c, miR-222, and miR-301b) were different between responders and nonresponders (Lin et al, 2014). The baseline levels or post-docetaxel change in the levels of 12 microRNAs (miR-20a, miR-20b, miR-21, miR-25, miR-132, miR-200a, miR-200b, miR-200c, miR-222, miR375 , miR-429, and miR-590-5p) were associated with overall survival (Lin et al, 2014). There was an overlap of four microRNAs (miR-20a, miR-200b, miR-200c, and miR-222) among these associations with docetaxel response and overall survival.

While our previous study was hypothesis-generating, the cornerstone of biomarker development is independent validation. Therefore, we performed a Phase 2 validation study on an independent cohort of CRPC patients treated with docetaxel to verify the association of these microRNAs with docetaxel response and overall survival. Here we report the findings of our study, which may have implications on CRPC therapy.

\section{PATIENTS AND METHODS}

Patients. The aim of this Phase 2 study was to validate the association of 14 circulating microRNAs with docetaxel response and overall survival in CRPC patients. The study was approved by relevant human research ethics committees (Approval number X14-0406), and was registered with the Australian New Zealand Clinical Trials Registry (ACTRN12607000077460). All participants provided written informed consent.

Patients with CRPC and commencing docetaxel chemotherapy were recruited from Calvary Mater Hospital, Royal Prince Alfred Hospital, Concord Repatriation General Hospital, Coffs Harbour Hospital, Westmead Hospital, Sydney Adventist Hospital and St George Hospital, from 2007 to 2015. CRPC was defined as prostate specific antigen (PSA) or clinical progression after maximal androgen blockade, with a minimum of 4 weeks between cessation of anti-androgens and commencement of docetaxel treatment.

PSA response after three cycles of docetaxel was used to classify chemoresponse, defined as follows: partial response, at least $50 \%$ decrease in PSA from baseline levels; progressive disease, increase of at least 25\% from baseline levels; stable disease, decrease of $<50 \%$ or an increase of $<25 \%$ from baseline levels (Scher et al, 2008). Patients with partial response were considered as responders, whereas those with stable or progressive disease were considered as non-responders.

Overall survival was defined as the time from initiation of the first cycle of docetaxel to the time of death or last follow-up. The survival status of the Phase 1 cohort was updated at the same time as the Phase 2 cohort for statistical analyses.

Blood collection. Blood samples were obtained from each patient at baseline before commencing docetaxel chemotherapy $\left(75 \mathrm{mg} \mathrm{m}^{-2}\right)$, and prior to the second cycle of treatment which is 3 weeks after the first dose (post-docetaxel). Blood samples were collected in BD Vacutainer tubes containing $\mathrm{K}_{2}$ EDTA, and centrifuged at $3000 \mathrm{~g}$ for $5 \mathrm{~min}$ at room temperature to separate the plasma. The plasma was aliquoted into cryovials and stored at $-80^{\circ} \mathrm{C}$

Quantitation of circulating microRNAs. Total RNA was extracted from $200 \mu \mathrm{l}$ of plasma using the miRNeasy Micro Kit (Qiagen, Germany) and eluted in $14 \mu \mathrm{l}$ of RNAse-free water as described previously except that ath-miR-159a was not added as a spike-in control (Lin et al, 2014). Total RNA of $4 \mu \mathrm{l}$ was used for reverse transcription into $15 \mu \mathrm{l}$ of cDNA, of which $8.75 \mu \mathrm{l}$ was preamplified into $25 \mu \mathrm{l}$ according to the protocol for Custom Taqman Array microRNA cards (Applied Biosystems, Foster City, CA, USA) as described previously, except that custom RT and pre-amplification primer pools of 17 microRNAs were used in the reactions (Applied Biosystems User Bulletin 4465407; Lin et al, 2014). The 17 microRNAs were the 14 potential biomarkers (miR-20a, miR20b, miR-21, miR-25, miR-132, miR-145a, miR-200a, miR-200b, miR200c, miR-222, miR-301b, miR-375, miR-429, and miR-590-5p) and 3 normalisers (miR-152, miR-30c, and miR-24). The pre-amplified cDNA $(25 \mu \mathrm{l})$ was diluted $1: 4$ with $0.1 \times$ TE buffer. Real-time PCR (RT-PCR) was performed on the diluted cDNA using individual Taqman microRNA assays (Applied Biosystems) with $2 \mu \mathrm{l} \mathrm{cDNA}$ per $10 \mu \mathrm{l}$ reaction volume according to the assay instructions, in duplicate, on the Applied Biosystems 7900HT system.

Data normalisation. The threshold for $C_{\mathrm{T}}$ values (number of cycles required for fluorescent signal to cross the set threshold) was set at 0.2 for all microRNAs. $C_{\mathrm{T}}$ values $>35$ were considered as non- 


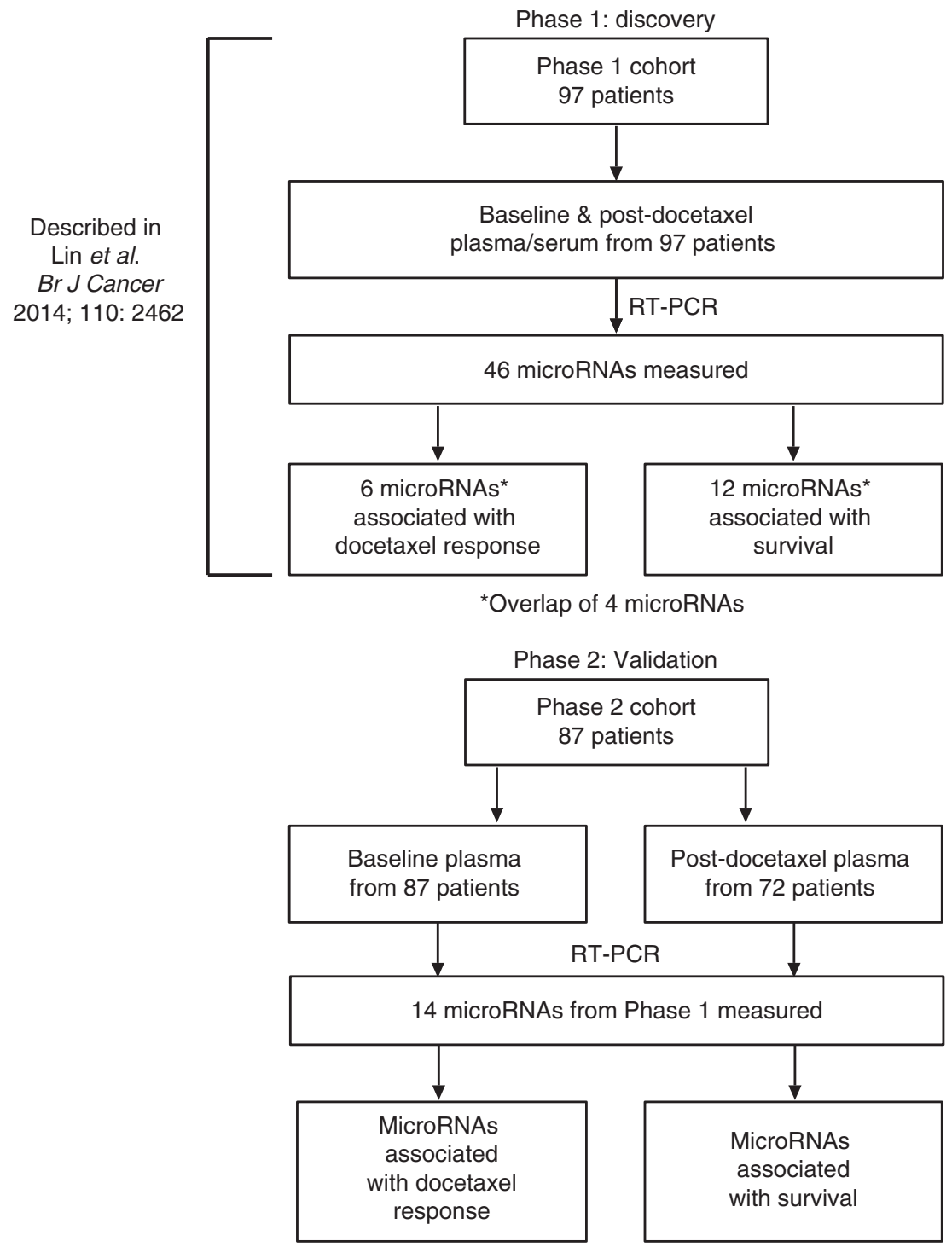

Figure 1. Phase 1 and Phase 2 study profiles.

specific or undetected and were removed. These missing values were replaced with the lowest value of the microRNA subtracted by 2 after normalisation. Previously in the Phase 1 study, we showed that normalisation with the global mean was better than with ath-miR159a spike-in or endogenous small nuclear RNA U6 (Lin et al, 2014). In the Phase 1 study, the circulating levels of miR-24, miR$30 \mathrm{c}$, and miR-152 was found to be highly correlated with the global mean of microRNAs used for normalisation, and thus the average of these three microRNAs was used as a surrogate of the global mean for normalising in this Phase 2 study (Supplementary Figure S1). These three microRNAs were not associated with chemoresponse or overall survival in the Phase 1 study. Normalisation was performed, by subtracting the average $\mathrm{C}_{\mathrm{T}}$ of $\mathrm{miR}-24, \mathrm{miR}-30 \mathrm{c}$, and $\mathrm{miR}-152$ from the $C_{\mathrm{T}}$ of every microRNA of each sample. The result (delta $C_{\mathrm{T}}$ ) was multiplied by -1 , where the normalised value can be interpreted as logarithm-twofold difference of the microRNA level relative to the average of the normalisers.

Statistical analyses. The difference in baseline microRNA levels or the post-docetaxel change in their levels, between responders and non-responders were compared by Mann-Whitney $U$ analysis using the R package 'multtest' (version 2.24.0). Survival analysis, Cox regression and crosstabulation analyses were performed using IBM SPSS Statistics (version 23.0.0.0).
Latent class analysis (LCA) was performed using the R package 'poLCA' (version 1.4.1) on R software version 3.2.1 (Linzer and Lewis, 2011) to identify risk groups. The analysis was performed on the median-centered normalised baseline levels of miR-132, miR-200a, miR-200b, miR-200c, and miR-375 of the Phase 1 cohort. These levels were categorised into quartiles for the analysis. The minimum Bayesian Information criterion was used to determine the most parsimonious number of latent classes.

Heatmap of microRNA levels was constructed using HeatMapViewer in GenePattern (version 3.2.3). T-test analysis was performed using the R package 'multtest' (version 2.24.0).

\section{RESULTS}

Characteristics of patients. For the Phase 2 study, plasma samples were obtained from an independent cohort of 87 patients with CRPC who commenced docetaxel chemotherapy from 2007 to 2015 (Table 1, Figure 1). Baseline plasma samples were obtained from all patients, whereas post-docetaxel samples were only available from 72 patients.

The Phase 2 cohort is a more contemporary cohort than Phase 1 as majority of Phase 2 patients (85\%) commenced docetaxel 
Table 1. Characteristics of Phase 1 and 2 cohorts

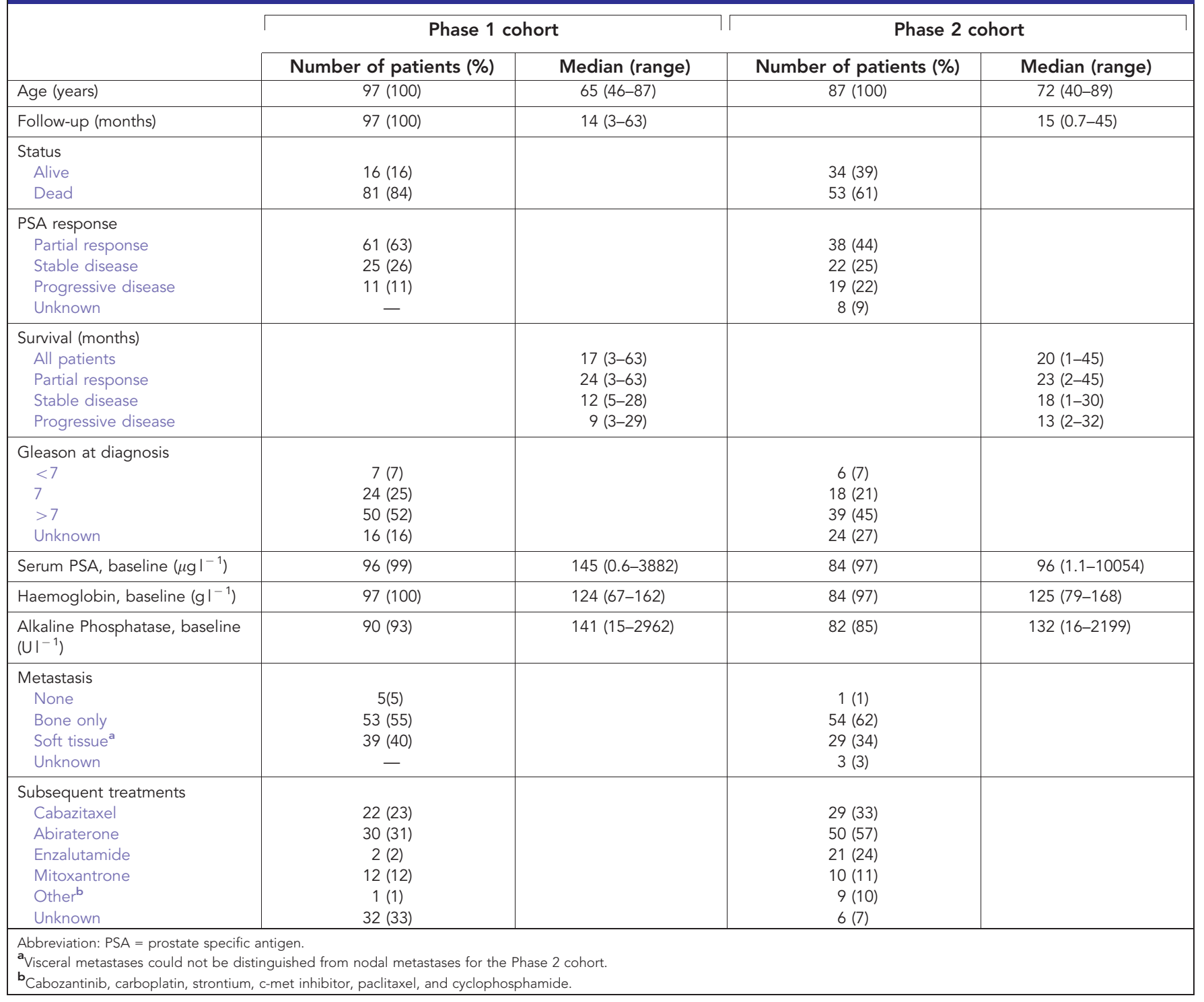

chemotherapy from 2012 onwards, whereas the Phase 1 cohort commenced docetaxel chemotherapy prior to 2012. A higher proportion of Phase 2 patients received abiraterone and enzalutamide treatments subsequent to docetaxel than Phase 1 patients (Table 1). Subsequent treatments were unknown for $30 \%$ of the Phase 1 cohort, but they were unlikely to receive abiraterone, enzalutamide or cabazitaxel, as they began docetaxel treatment from 2005 to 2007, prior to the clinical availability of these therapeutics in Australia.

The Phase 2 cohort had a lower proportion of responders to docetaxel, and a lower median for PSA levels compared to the Phase 1 cohort (Table 1). The number of deaths was higher for the Phase 1 cohort (84\%) after the survival status for 26 patients was updated since the last report of the study (Lin et al, 2014), compared to the Phase 2 cohort (61\%).

Detection of microRNAs. In the Phase 1 study, six microRNAs were associated with docetaxel response and 12 microRNAs were associated with overall survival. There was an overlap of four microRNAs among these associations, hence a total of 14 microRNAs was investigated in the Phase 2 study (Figure 1). These microRNAs and the three normalisers (miR-24, miR-30c, and miR-152) were detected in at least $95 \%$ of the plasma samples (151/159), except for miR-429 which was only detected in $79 \%$ of the plasma samples (125/159). The median number of RT-PCR cycles required to cross the set fluorescence threshold $\left(C_{\mathrm{T}}\right)$ ranged from 16.9 to 27 for all microRNAs except for miR-429, which was 29.4 (Figure 2), indicating that the microRNAs could be detected and quantitated reliably, and that the levels of miR-429 were generally low. The levels of each microRNA was normalised to the average of the three normalisers (further details in method) for statistical analyses.

Circulating microRNAs and chemoresponse of Phase 2 cohort. Previously in the Phase 1 study, we had identified that the baseline levels of miR-146a, miR-200b, and miR-200c; and the postdocetaxel change in the levels of miR-20a, miR-222, and miR-301b, were significantly different between responders and non-responders (Lin et al, 2014). In the Phase 2 cohort, the differences in these microRNAs were not significant between responders and nonresponders $(P \geqslant 0.39)$ except for the post-docetaxel change in miR$301 \mathrm{~b}(P=0.04$, Figure $3 \mathrm{~A})$. However, the difference in miR-301b was the reverse of that observed for the Phase 1 cohort (Figure 3A) and thus was not considered to be associated with chemoresponse. 


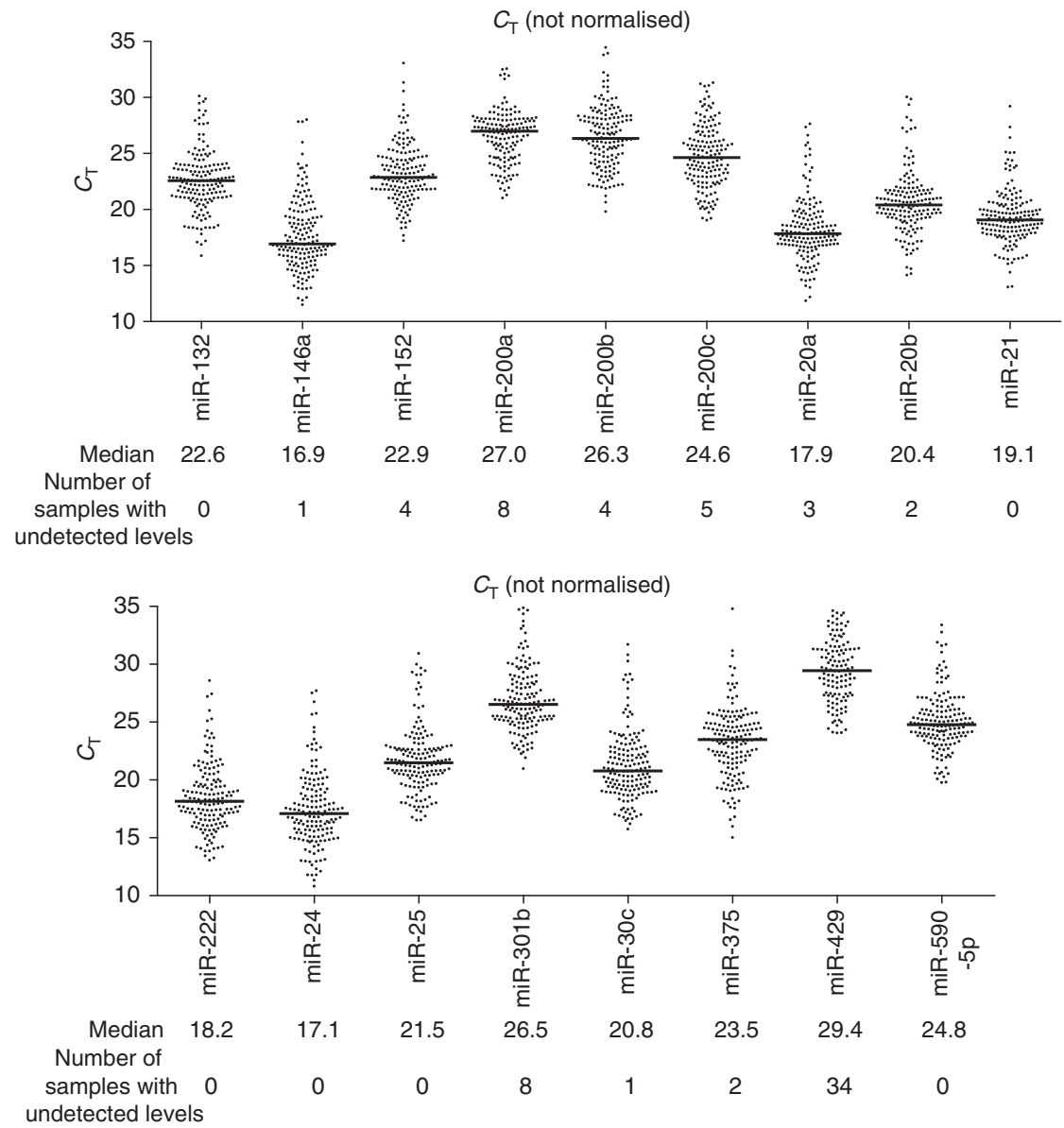

Figure 2. Dot plots of un-normalised $C_{T}$ values of circulating microRNAs in baseline and post-chemo plasma samples of the Phase 2 cohort. Each data point represents a plasma sample. $C_{T}$, number of cycles for fluorescent signal to cross the set threshold.

Circulating microRNAs and overall survival of Phase 2 cohort. In the Phase 1 study, we had also identified that the baseline levels of miR-20a, miR-21, miR-132, miR-200a, miR-200b, miR-200c, miR-375, miR-429, and miR-590-5p; and the post-docetaxel change in the levels of miR-20a, miR-20b, miR-25, miR-132, and miR-222, were associated with overall survival (Lin et al, 2014). Both the baseline levels and post-docetaxel change in levels for two of these microRNAs (miR-20a and miR-132) were associated with overall survival in that study.

In the Phase 2 cohort, only the baseline levels of six microRNAs were associated with overall survival according to both log-rank test of patients dichotomised by median levels, and cox regression analysis of microRNAs as continuous variables. These six microRNAs were miR-132, miR-200a, miR-200b, miR-200c, miR-375, and miR-429 (log-rank $P$ range from $6 \times 10^{-6}$ to $2 \times 10^{-2}$, Figure $3 \mathrm{~B}$ ). Higher baseline levels of these microRNAs were associated with shorter overall survival.

Prognostic microRNA signature. Validation of the association of the microRNAs with overall survival instead of chemoresponse indicates that the circulating microRNAs are predominantly prognostic. To identify patient risk groups based on the combination of the six prognostic microRNAs (miR-132, miR200a, miR-200b, miR-200c, miR-375, and miR-429), LCA was performed on the baseline levels of these microRNAs for the Phase 1 cohort. MiR-429 was excluded from LCA as its levels were undetected in majority of the samples and could not be categorised for the analysis.
LCA, which is an unsupervised approach, grouped the patients into two subgroups, referred to as Class 1 and Class 2. The overall survival of Class 1 patients was significantly shorter than Class 2 patients (median 12 vs 23.9 months, log-rank $P=0.0000001$; HR 3.45, 95\% CI 2.12-5.62, $P=0.000001$; Figure $4 \mathrm{~A})$. The baseline levels of all six microRNAs were significantly different between Class 1 and Class 2 patients (adjusted $P \leq 0.0007$; Figure 4B).

An optimal cutpoint for the sum of the baseline levels of all six microRNAs that could distinguish between high- and low-risk patients were determined from the LCA groupings (Figure 4C). Patients were considered to be high risk if the sum of the baseline levels of all six microRNAs, expressed as median-centered log2 values, was greater than zero. With this cutpoint, 35 out of the 97 patients of the Phase 1 cohort have this high-risk signature, where their overall survival was significantly shorter (11 vs 23.7 months, $P=0.000001 ; \quad$ HR $3.43, \quad 95 \%$ CI $2.10-5.57, \quad P=0.000001$; Figure 4D). In the Phase 2 cohort, 40 out of the 87 patients have the high-risk signature and their overall survival was significantly shorter (median 12.9 vs 30.5 months, log-rank $P=0.000001$; HR 4.12, 95\% CI 2.20-7.70, $P=0.00001$; Figure 4E).

\section{Comparison of microRNA signature with clinicopathological} parameters. High alkaline phosphatase and PSA levels, low haemoglobin levels, and the presence of visceral metastases are clinicopathological factors known to be associated with poorer prognosis in CRPC patients (Halabi et al, 2014). Information on these factors is available for both of our cohorts, except for visceral 
A
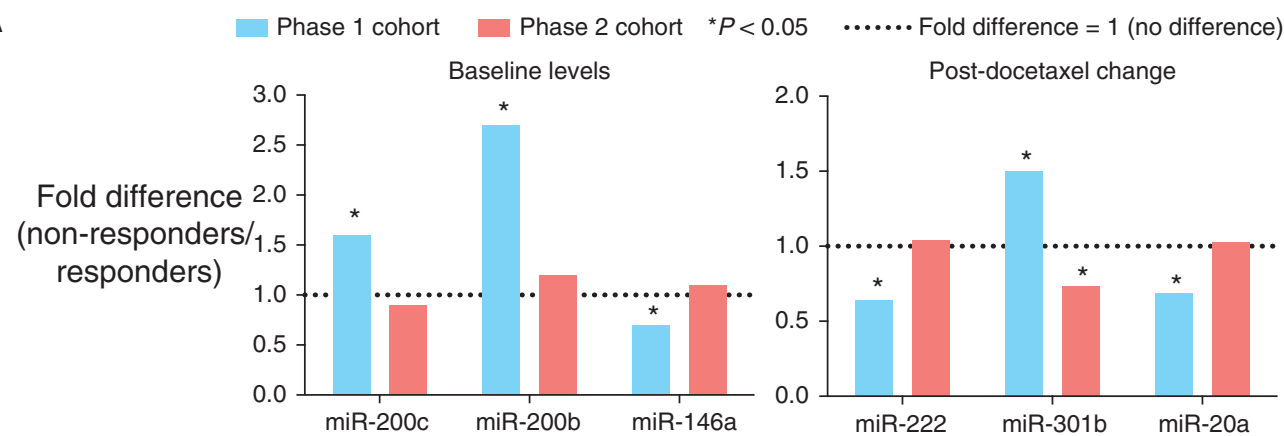

B

Phase 2 cohort

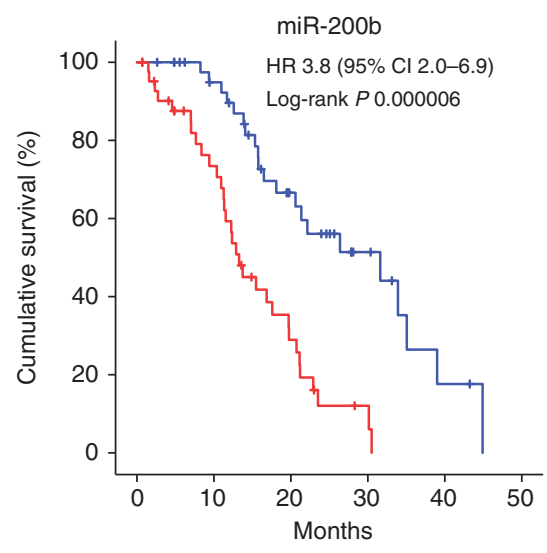

— Low levels ( $\leq$ median) — High levels (>median)
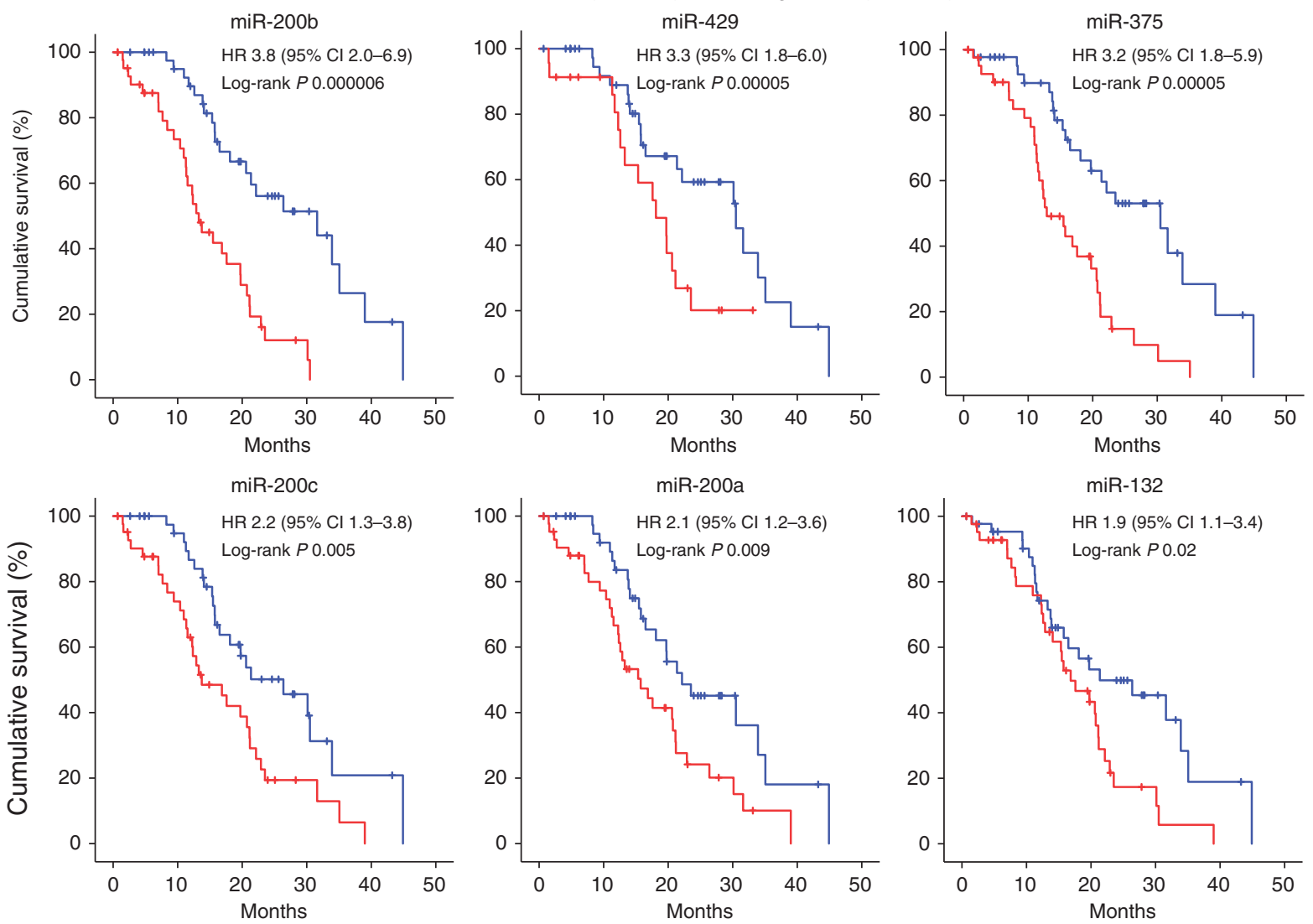

Figure 3. Validation of circulating microRNAs in Phase 2 cohort. (A) Comparison of circulating microRNA levels between docetaxel responders and non-responders in Phase 1 and 2 cohorts (Mann-Whitney U-test analysis); (B) Survival curves of Phase 2 cohort, dichotomised according to the median baseline microRNA level. Abbreviations: $\mathrm{Cl}$, confidence interval; $\mathrm{HR}$, hazard ratio for dichotomised groups.

metastases, which could not be distinguished from nodal metastases in the Phase 2 cohort database.

A significantly higher proportion of patients with the high-risk microRNA signature have elevated levels of alkaline phosphatase and serum PSA, and low levels of haemoglobin, for both Phase 1 and 2 cohorts (Supplementary Table S1, $P \leqslant 0.02$ ). However, multivariable cox regression of the microRNA signature with these clinicopathological factors in relation to overall survival revealed that only the microRNA signature was an independent factor in both cohorts (Table 2). Haemoglobin level was an independent factor in the Phase 1 cohort only (Table 2).

\section{DISCUSSION}

In this Phase 2 study of circulating microRNAs in CRPC, we confirmed that six microRNAs were associated with overall survival - miR-132, miR-200a, miR-200b, miR-200c, miR-375, and miR-429. Higher levels of these microRNAs were associated with poorer survival outcome. We derived a prognostic microRNA signature consisting of the sum of the levels of these six microRNAs, which was also validated in the Phase 2 cohort.

The association of these six microRNAs with overall survival of CRPC indicates that these microRNAs may be involved in the pathogenesis and/or progression of metastatic prostate cancer. However, the mechanism of action of these microRNAs is difficult to elucidate as the cellular source of the microRNAs is currently unknown. The microRNAs could be produced by cancer cells or non-malignant cells, and released into circulation encapsulated in extracellular vesicles (microvesicles, endosomes, and apoptotic bodies) or bound to Argonaute proteins (Turchinovich et al, 2013; Fujita et al, 2016).

Many studies have indicated that circulating microRNAs are a form of cell-cell communication, as they can be taken up by 
A

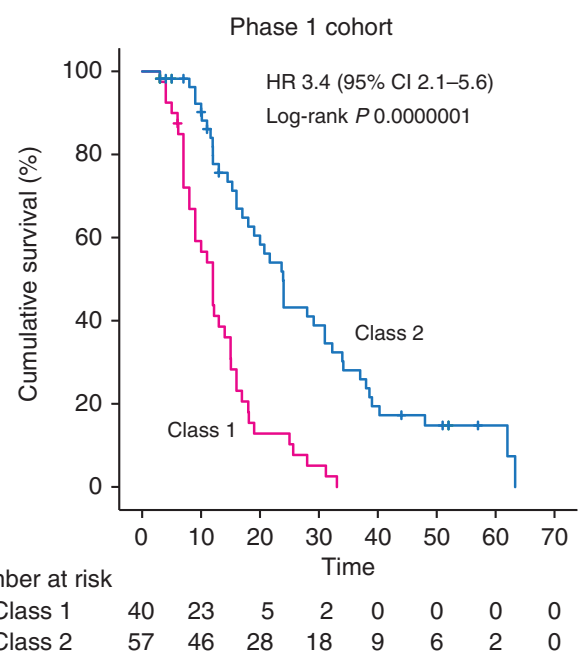

C

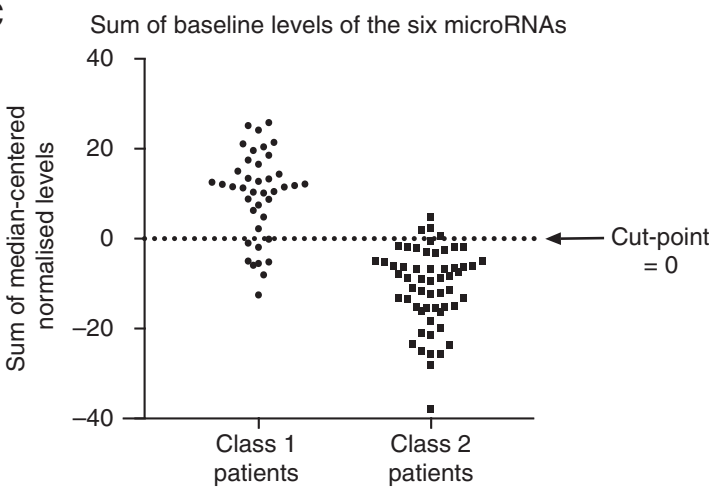

D

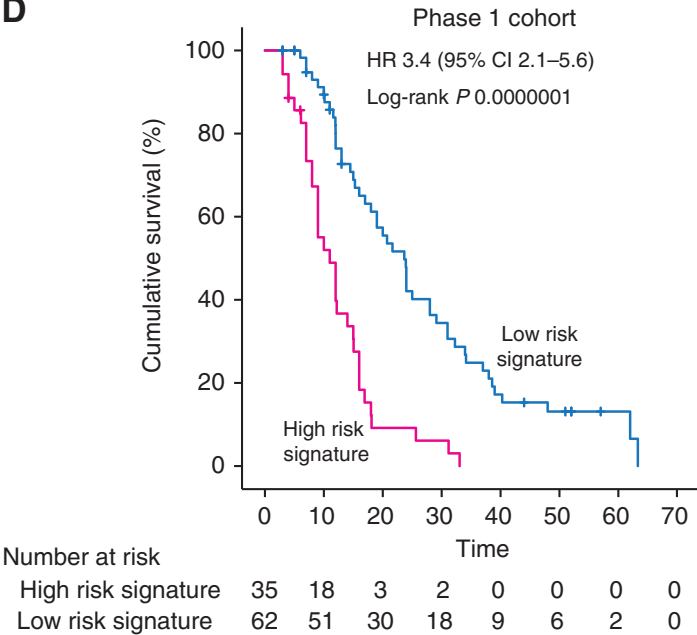

B

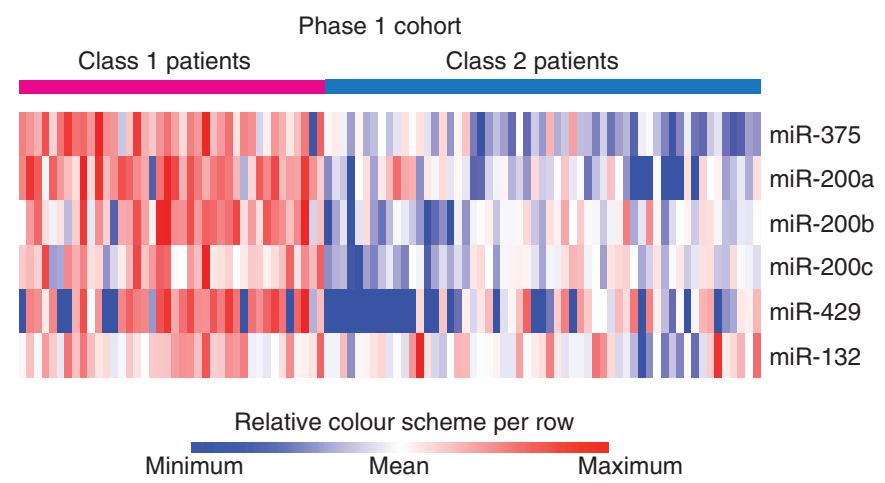

microRNA signature

High risk: miR-200a + miR-200b + miR-200c

$+m i R-429+m i R-375+m i R-132>0$

Low risk: miR-200a + miR-200b + miR-200c

$+m i R-429+m i R-375+m i R-132 \leq 0$

E

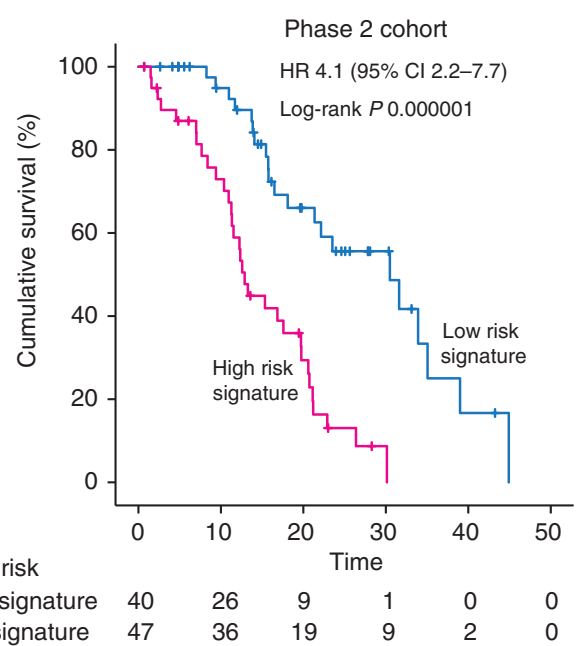

Figure 4. Derivation of prognostic microRNA signature. (A) Survival curves of Phase 1 cohort, grouped by LCA of baseline microRNA levels; (B) Heatmap of baseline levels of the six microRNAs for Phase 1 cohort. These levels were significantly different between Class 1 and Class 2 patients (t-test Benjamini-Hochberg adjusted $P \leqslant 0.0007$ ); (C) Determination of cutpoint to define risk groups for prognostic microRNA signature. The microRNA signature is the sum of the median-centered normalised baseline levels of six microRNAs; (D) Survival curves of Phase 1 cohort, grouped according to the microRNA signature; (E) Survival curves of Phase 2 cohort, grouped according to the microRNA signature.

recipient cells and exert functional effects on the recipient cells such as proliferation, invasion, and angiogenesis (Turchinovich et al, 2013; Fujita et al, 2016). Exosomal-containing microRNAs from cancer cells were shown to promote pre-metastatic niches in other organs by modulating the stromal cells of the metastatic site (Rana et al, 2013). It is believed that secretion of microRNAs in extracellular vesicles is a selective process, as the profiles of secreted microRNAs do not match intracellular profiles (Collino et al, 2010; Mittelbrunn et al, 2011). On the other hand, some circulating
microRNAs may be by-products of dead cells in the form of apoptotic bodies (Turchinovich et al, 2011), or simply nonselectively released by cells as AGO-bound complexes (Turchinovich et al, 2013), and thus may be a reflection of tumour burden.

Interestingly four of the six microRNAs associated with overall survival - miR-200a, miR-200b, miR-200c, and miR-429, are members of the miR-200 family. These microRNAs are wellknown for the regulation of epithelial-to-mesenchymal transition 
Table 2. Cox regression analyses of microRNA signature and clinicopathological factors in relation to overall survival

\begin{tabular}{|c|c|c|c|c|c|c|}
\hline Variables $^{a}$ & Cases & Events & \multicolumn{2}{|c|}{ Univariable Cox regression } & \multicolumn{2}{|c|}{ Multivariable Cox regression } \\
\hline \multicolumn{7}{|l|}{ Phase 1} \\
\hline Haemoglobin & 97 & 81 & $0.98(0.97-0.99)$ & 0.00007 & $0.98(0.97-0.99)$ & 0.001 \\
\hline Alkaline phosphatase & 90 & 76 & $1.00(1.00-1.00)$ & 0.03 & $1.00(1.00-1.00)$ & 0.43 \\
\hline Serum PSA & 96 & 80 & $1.00(1.00-1.00)$ & 0.14 & $1.00(1.00-1.00)$ & 0.18 \\
\hline Haemoglobin & 82 & 52 & $0.97(0.95-0.99)$ & 0.001 & $0.98(0.96-1.00)$ & 0.06 \\
\hline Alkaline phosphatase & 80 & 52 & $1.00(1.00-1.00)$ & 0.05 & $1.00(1.00-1.00)$ & 0.85 \\
\hline Serum PSA & 82 & 52 & $1.00(1.00-1.00)$ & 0.45 & $1.00(1.00-1.00)$ & 0.6 \\
\hline
\end{tabular}

(EMT) where low levels of these microRNAs induced EMT through the upregulation of their target, ZEB1, an EMT transcription factor (Gregory et al, 2008). Recent studies showed that high levels of miR-200 microRNAs can promote metastasis of breast cancer, as transfer of exosomes containing miR-200 microRNAs from metastatic to non-metastatic breast cancer cells promoted metastatic colonisation of the latter in mouse models, presumably through the reverse of EMT, mesenchymal-toepithelial transition (MET; Le et al, 2014). Another proposed mechanism of metastasis is the regulation of the tumour secretome by SEC23A, another target of miR-200 microRNAs (Korpal et al, 2011). High levels of circulating miR-200 microRNAs were associated with poor prognosis in breast cancer (Korpal et al, 2011), similar to our observation for CRPC, thus similar mechanisms could be involved. Higher levels of miR-200b have been found in prostate carcinoma compared to matched normal tissue (Hart et al, 2013), suggesting that miR-200 microRNAs could be produced by the prostate cancer cells to enhance their metastatic colonisation.

The levels of the other prognostic microRNA, miR-375, were also higher in prostate carcinoma compared to matched normal tissue (Hart et al, 2013). Circulating levels of miR-375 were higher in prostate cancer patients with metastases than those without (Bryant et al, 2012; Nguyen et al, 2012). Plasma exosomal miR-375 showed prognostic value in a screening cohort of 23 CRPC patients (Huang et al, 2014). The function of miR-375 in CRPC pathogenesis may be similar to that of the miR-200 family as SEC23A is also a target of miR-375, and ectopic expression of miR375 promoted MET in prostate cancer cells (Szczyrba et al, 2011; Selth et al, 2016). Unlike the miR-200 family, MET regulation by miR-375 is presumably through its target, YAP1, a transcription factor (Selth et al, 2016). ZEB1 was shown to repress the expression of miR-375 (Selth et al, 2016), thus downregulation of ZEB1 expression by miR-200 family members may contribute to upregulation of miR-375.

The other microRNA associated with CRPC prognosis in our study is miR-132. Several studies indicate that it has a tumour suppressive role depending on the cancer type, where overexpression of miR-132 in prostate, breast, ovarian, lung or colorectal cancer cell lines inhibited growth, migration or invasion (Luo et al, 2014; Zhang et al, 2014; Li et al, 2015; Fu et al, 2016; Qu et al, 2016; Tian et al, 2016). Its expression was also downregulated in prostate, breast or ovarian tumours compared to benign tissue (Damavandi et al, 2016; Fu et al, 2016; Qu et al, 2016; Tian et al, 2016). Therefore, the upregulated levels of circulating miR-132 in CRPC patients with poorer outcome in our study are unlikely to be derived from prostate cancer cells. Instead, miR-132 may be produced by immune cells as it is involved in the regulation of innate immunity, where expression of miR-132 in monocytes and macrophages is induced by bacterial/viral ligands (Taganov et al, 2006; Lagos et al, 2010; Wanet et al, 2012). Peripheral blood mononuclear cells of patients with rheumatoid arthritis have a higher expression of miR-132 than healthy individuals (Pauley et al, 2008). Therefore miR-132 may be indirectly enhancing cancer cell growth through the promotion of inflammation. In addition, miR-132 may also be enhancing angiogenesis in tumours as it was shown to be highly expressed in the endothelium of tumours and its ectopic expression in endothelial cells increased their proliferation and tube-forming capacity through the suppression of its target p120RasGAP (Anand and Cheresh, 2011).

A better understanding on the function of the circulating microRNAs may lead to new therapeutic strategies to improve patient outcome. If these microRNAs are influencing tumour growth, suppression of their function using microRNA-based therapeutics such as synthetic anti-miR oligonucleotides may improve patient outcome (van Rooij and Kauppinen, 2014). In mice models, exosome-containing miR-200 microRNAs from metastatic breast cancer cells could not confer metastatic ability to non-metastatic breast cancer cells treated with anti-miR-200 oligonucleotides, demonstrating suppression of miR-200 function by the anti-miRs (Le et al, 2014). Development of microRNAbased therapeutics is ongoing with recent advances highlighted by Miravirsen (inhibitor of miR-34) reaching Phase 2 clinical trials for treating hepatitis C (Janssen et al, 2013; van Rooij and Kauppinen, 2014). A few other microRNA inhibitors are in Phase 1 clinical trials for liver cancer or hepatitis C, while others are in preclinical stages (van Rooij and Kauppinen, 2014). However the risk of suppressing the miR-200 microRNAs or miR-375 is the promotion of EMT resulting in further metastases, as downregulation of these microRNAs promote EMT as described above. Therefore, further research is required on the feasibility of targeting these microRNA family, such as focusing on their gene targets instead. MiR-132 may be a better option for microRNAbased therapeutics, as its effects are likely to be on immune or endothelial cells which indirectly influence tumour growth as discussed above.

A limitation of our study is that lactase dehydrogenase and ECOG performance status data were not available for our cohorts. These data are required for estimating CRPC prognosis with the Halabi nomogram (Halabi et al, 2013), and thus the prognostic performance of our microRNAs could not be compared to the Halabi nomogram. Another limitation is the unknown source of the plasma microRNAs, as it is unclear whether circulating microRNAs originate from the cancer or the host as a reaction to 
the cancer. The source of the microRNAs may provide insight into their biology and mechanism of action.

Overall, the findings from this Phase 2 study highlight the importance of validating potential biomarkers as not all findings were reproducible. The value of circulating microRNAs as prognostic biomarkers has been confirmed. Further research is required to understand the role of these microRNAs and to validate the prognostic microRNA signature in a Phase 3 study.

\section{ACKNOWLEDGEMENTS}

We gratefully acknowledge the patients, and the nurses and investigators of the PRIMe consortium. This study was funded by Cancer Institute New South Wales (10/TPG/1-04). Cancer Council New South Wales funded PRIMe (PG 10-01) which coordinated the collection of plasma samples and clinical information. Dr Kate Mahon is funded by a Movember Clinician Scientist Award awarded through Prostate Cancer Foundation of Australia's Research Program.

\section{CONFLICT OF INTEREST}

The authors declare no conflict of interest.

\section{REFERENCES}

Anand S, Cheresh DA (2011) MicroRNA-mediated regulation of the angiogenic switch. Curr Opin Hematol 18(3): 171-176.

Bishr M, Saad F (2013) Overview of the latest treatments for castrationresistant prostate cancer. Nat Rev Urol 10: 522-528.

Bryant RJ, Pawlowski T, Catto JW, Marsden G, Vessella RL, Rhees B, Kuslich C, Visakorpi T, Hamdy FC (2012) Changes in circulating microRNA levels associated with prostate cancer. Br J Cancer 106(4): 768-774.

Cheng HH, Mitchell PS, Kroh EM, Dowell AE, Chery L, Siddiqui J, Nelson PS, Vessella RL, Knudsen BS, Chinnaiyan AM, Pienta KJ, Morrissey C, Tewari M (2013) Circulating microRNA profiling identifies a subset of metastatic prostate cancer patients with evidence of cancer-associated hypoxia. PLoS ONE 8(7): e69239.

Collino F, Deregibus MC, Bruno S, Sterpone L, Aghemo G, Viltono L, Tetta C, Camussi G (2010) Microvesicles derived from adult human bone marrow and tissue specific mesenchymal stem cells shuttle selected pattern of miRNAs. PLoS ONE 5(7): e11803.

Damavandi Z, Torkashvand S, Vasei M, Soltani BM, Tavallaei M, Mowla SJ (2016) Aberrant expression of breast development-related microRNAs, miR-22, miR-132, and miR-212, in breast tumor tissues. J Breast Cancer 19(2): 148-155.

Fabris L, Ceder Y, Chinnaiyan AM, Jenster GW, Sorensen KD, Tomlins S, Visakorpi T, Calin GA (2016) The potential of microRNAs as prostate cancer biomarkers. Eur Urol 70(2): 312-322.

Fendler A, Stephan C, Yousef GM, Kristiansen G, Jung K (2016) The translational potential of microRNAs as biofluid markers of urological tumours. Nat Rev Urol 13(12): 734-752.

Ferraldeschi R, Welti J, Luo J, Attard G, de Bono JS (2015) Targeting the androgen receptor pathway in castration-resistant prostate cancer: progresses and prospects. Oncogene 34(14): 1745-1757.

Fu W, Tao T, Qi M, Wang L, Hu J, Li X, Xing N, Du R, Han B (2016) MicroRNA-132/212 upregulation inhibits TGF-beta-mediated epithelialmesenchymal transition of prostate cancer cells by targeting SOX4. Prostate 76(16): 1560-1570.

Fujita Y, Yoshioka Y, Ochiya T (2016) Extracellular vesicle transfer of cancer pathogenic components. Cancer Sci 107(4): 385-390.

Gonzales JC, Fink LM, Goodman Jr. OB, Symanowski JT, Vogelzang NJ, Ward DC (2011) Comparison of circulating MicroRNA 141 to circulating tumor cells, lactate dehydrogenase, and prostate-specific antigen for determining treatment response in patients with metastatic prostate cancer. Clin Genitourin Cancer 9(1): 39-45.
Gregory PA, Bracken CP, Bert AG, Goodall GJ (2008) MicroRNAs as regulators of epithelial-mesenchymal transition. Cell Cycle 7(20): 3112-3118.

Halabi S, Lin CY, Kelly WK, Fizazi KS, Moul JW, Kaplan EB, Morris MJ, Small EJ (2014) Updated prognostic model for predicting overall survival in first-line chemotherapy for patients with metastatic castration-resistant prostate cancer. J Clin Oncol 32(7): 671-677.

Halabi S, Lin CY, Small EJ, Armstrong AJ, Kaplan EB, Petrylak D, Sternberg CN, Shen L, Oudard S, de Bono J, Sartor O (2013) Prognostic model predicting metastatic castration-resistant prostate cancer survival in men treated with second-line chemotherapy. J Natl Cancer Inst 105(22): 1729-1737.

Hart M, Nolte E, Wach S, Szczyrba J, Taubert H, Rau T, Hartmann A, Grasser FA, Wullich B (2013) Comparative microRNA profiling of prostate carcinomas with increasing tumor stage by deep-sequencing. Mol Cancer Res 12(2): 250-263.

Huang X, Yuan T, Liang M, Du M, Xia S, Dittmar R, Wang D, See W, Costello BA, Quevedo F, Tan W, Nandy D, Bevan GH, Longenbach S, Sun Z, Lu Y, Wang T, Thibodeau SN, Boardman L, Kohli M, Wang L (2014) Exosomal miR-1290 and miR-375 as prognostic markers in castration-resistant prostate cancer. Eur Urol 67(1): 33-41.

Janssen HL, Reesink HW, Lawitz EJ, Zeuzem S, Rodriguez-Torres M, Patel K, van der Meer AJ, Patick AK, Chen A, Zhou Y, Persson R, King BD, Kauppinen S, Levin AA, Hodges MR (2013) Treatment of HCV infection by targeting microRNA. N Engl J Med 368(18): 1685-1694.

Korpal M, Ell BJ, Buffa FM, Ibrahim T, Blanco MA, Celia-Terrassa T, Mercatali L, Khan Z, Goodarzi H, Hua Y, Wei Y, Hu G, Garcia BA, Ragoussis J, Amadori D, Harris AL, Kang Y (2011) Direct targeting of Sec23a by miR-200s influences cancer cell secretome and promotes metastatic colonization. Nat Med 17(9): 1101-1108.

Kozomara A, Griffiths-Jones S (2011) miRBase: integrating microRNA annotation and deep-sequencing data. Nucleic Acids Res 39(Database issue): D152-D157.

Lagos D, Pollara G, Henderson S, Gratrix F, Fabani M, Milne RS, Gotch F, Boshoff C (2010) miR-132 regulates antiviral innate immunity through suppression of the p300 transcriptional co-activator. Nat Cell Biol 12(5): 513-519.

Le MT, Hamar P, Guo C, Basar E, Perdigao-Henriques R, Balaj L, Lieberman J (2014) miR-200-containing extracellular vesicles promote breast cancer cell metastasis. J Clin Invest 124(12): 5109-5128.

Li Y, Zu L, Wang Y, Wang M, Chen P, Zhou Q (2015) miR-132 inhibits lung cancer cell migration and invasion by targeting SOX4. J Thorac Dis 7(9): $1563-1569$.

Lin HM, Castillo L, Mahon KL, Chiam K, Lee BY, Nguyen Q, Boyer MJ, Stockler MR, Pavlakis N, Marx G, Mallesara G, Gurney H, Clark SJ, Swarbrick A, Daly RJ, Horvath LG (2014) Circulating microRNAs are associated with docetaxel chemotherapy outcome in castration-resistant prostate cancer. Br J Cancer 110(10): 2462-2471.

Linzer DA, Lewis JB (2011) poLCA: an R Package for polytomous variable latent class analysis. J Stat Softw 42(10): 1-29.

Lujambio A, Lowe SW (2012) The microcosmos of cancer. Nature 482(7385): 347-355.

Luo J, Meng C, Tang Y, Zhang S, Wan M, Bi Y, Zhou X (2014) miR-132/212 cluster inhibits the growth of lung cancer xenografts in nude mice. Int $J$ Clin Exp Med 7(11): 4115-4122.

Mittelbrunn M, Gutierrez-Vazquez C, Villarroya-Beltri C, Gonzalez S, Sanchez-Cabo F, Gonzalez MA, Bernad A, Sanchez-Madrid F (2011) Unidirectional transfer of microRNA-loaded exosomes from $\mathrm{T}$ cells to antigen-presenting cells. Nat Commun 2: 282.

Nguyen HC, Xie W, Yang M, Hsieh CL, Drouin S, Lee GS, Kantoff PW (2012) Expression differences of circulating microRNAs in metastatic castration resistant prostate cancer and low-risk, localized prostate cancer. Prostate 73(4): 346-354.

Pauley KM, Satoh M, Chan AL, Bubb MR, Reeves WH, Chan EK (2008) Upregulated miR-146a expression in peripheral blood mononuclear cells from rheumatoid arthritis patients. Arthritis Res Ther 10(4): R101.

Petrylak DP, Tangen CM, Hussain MH, Lara Jr. PN, Jones JA, Taplin ME, Burch PA, Berry D, Moinpour C, Kohli M, Benson MC, Small EJ, Raghavan D, Crawford ED (2004) Docetaxel and estramustine compared with mitoxantrone and prednisone for advanced refractory prostate cancer. N Engl J Med 351(15): 1513-1520.

Qu W, Ding SM, Cao G, Wang SJ, Zheng XH, Li GH (2016) miR-132 mediates a metabolic shift in prostate cancer cells by targeting Glut1. FEBS Open Bio 6(7): 735-741. 
Rana S, Malinowska K, Zoller M (2013) Exosomal tumor microRNA modulates premetastatic organ cells. Neoplasia 15(3): 281-295.

Scher HI, Halabi S, Tannock I, Morris M, Sternberg CN, Carducci MA, Eisenberger MA, Higano C, Bubley GJ, Dreicer R, Petrylak D, Kantoff P, Basch E, Kelly WK, Figg WD, Small EJ, Beer TM, Wilding G, Martin A, Hussain M (2008) Design and end points of clinical trials for patients with progressive prostate cancer and castrate levels of testosterone: recommendations of the Prostate Cancer Clinical Trials Working Group. $J$ Clin Oncol 26(7): 1148-1159.

Selth LA, Das R, Townley SL, Coutinho I, Hanson AR, Centenera MM, Stylianou N, Sweeney K, Soekmadji C, Jovanovic L, Nelson CC, Zoubeidi A, Butler LM, Goodall GJ, Hollier BG, Gregory PA, Tilley WD (2016) A ZEB1-miR-375-YAP1 pathway regulates epithelial plasticity in prostate cancer. Oncogene 36(1): 24-34.

Sita-Lumsden A, Dart DA, Waxman J, Bevan CL (2013) Circulating microRNAs as potential new biomarkers for prostate cancer. Br J Cancer 108(10): 1925-1930.

Szczyrba J, Nolte E, Wach S, Kremmer E, Stohr R, Hartmann A, Wieland W, Wullich B, Grasser FA (2011) Downregulation of Sec23A protein by miRNA-375 in prostate carcinoma. Mol Cancer Res 9(6): 791-800.

Taganov KD, Boldin MP, Chang KJ, Baltimore D (2006) NF-kappaB-dependent induction of microRNA miR-146, an inhibitor targeted to signaling proteins of innate immune responses. Proc Natl Acad Sci USA 103(33): 12481-12486.

Tannock IF, de Wit R, Berry WR, Horti J, Pluzanska A, Chi KN, Oudard S, Theodore C, James ND, Turesson I, Rosenthal MA, Eisenberger MA (2004) Docetaxel plus prednisone or mitoxantrone plus prednisone for advanced prostate cancer. N Engl J Med 351(15): 1502-1512.

Thieu W, Tilki D, deVere White RW, Evans CP (2014) The role of microRNA in castration-resistant prostate cancer. Urol Oncol 32(5): 517-523.

Tian H, Hou L, Xiong YM, Huang JX, Zhang WH, Pan YY, Song XR (2016) miR-132 targeting E2F5 suppresses cell proliferation, invasion, migration in ovarian cancer cells. Am J Transl Res 8(3): 1492-1501.
Torre LA, Bray F, Siegel RL, Ferlay J, Lortet-Tieulent J, Jemal A (2015) Global cancer statistics, 2012. CA Cancer J Clin 65(2): 87-108.

Turchinovich A, Samatov TR, Tonevitsky AG, Burwinkel B (2013) Circulating miRNAs: cell-cell communication function? Front Genet 4: 119.

Turchinovich A, Weiz L, Langheinz A, Burwinkel B (2011) Characterization of extracellular circulating microRNA. Nucleic Acids Res 39(16): $7223-7233$.

van Rooij E, Kauppinen S (2014) Development of microRNA therapeutics is coming of age. EMBO Mol Med 6(7): 851-864.

Wanet A, Tacheny A, Arnould T, Renard P (2012) miR-212/132 expression and functions: within and beyond the neuronal compartment. Nucleic Acids Res 40(11): 4742-4753.

Wong YN, Ferraldeschi R, Attard G, de Bono J (2014) Evolution of androgen receptor targeted therapy for advanced prostate cancer. Nat Rev Clin Oncol 11(6): 365-376.

Zhang HL, Yang LF, Zhu Y, Yao XD, Zhang SL, Dai B, Zhu YP, Shen YJ, Shi GH, Ye DW (2011) Serum miRNA-21: elevated levels in patients with metastatic hormone-refractory prostate cancer and potential predictive factor for the efficacy of docetaxel-based chemotherapy. Prostate 71(3): 326-331.

Zhang ZG, Chen WX, Wu YH, Liang HF, Zhang BX (2014) MiR-132 prohibits proliferation, invasion, migration, and metastasis in breast cancer by targeting HN1. Biochem Biophys Res Commun 454(1): 109114 .

This work is published under the standard license to publish agreement. After 12 months the work will become freely available and the license terms will switch to a Creative Commons AttributionNonCommercial-Share Alike 4.0 Unported License.

Supplementary Information accompanies this paper on British Journal of Cancer website (http://www.nature.com/bjc) 